\title{
LA LEGACIÓN DE FRANCESCO BARBERINI EN ESPAÑA: UNOS RETRATOS PARA EL CARDENAL Y UN BREVE PONTIFICIO PARA DIEGO VELÁZQUEZ, «CLERICO CONIUGATO»*
}

\author{
POR \\ SILVANO GIORDANO \\ Universidad Pontificia Gregoriana \\ $y$ \\ SALVADOR SALORT PONS \\ Universidad Complutense
}

\begin{abstract}
Se presenta nueva documentación encontrada en el Archivo Secreto Vaticano sobre el envío — desde Madrid a Roma - de algunos retratos realizados por Velázquez para el cardenal Francisco Barberini. Entre éstos un dibujo preparatorio para una imagen del Conde Duque, que el sevillano tenía que pintar según expresas indicaciones dejadas por el cardenal y su secretario Cassiano dal Pozzo en Madrid, en 1626. Asimismo se estudia el breve pontificio concedido en ese año a Velázquez, gracias a la gestión de Barberini ante el Papa, y por el que el pintor pudo disfrutar de un beneficio eclesiástico aun siendo un laico casado y con hijos. Como veremos, todas estas noticias pondrán de manifiesto el interés del Conde Duque por la carrera del pintor, el extraordinario trato de favor que éste recibía durante sus primeros años en el Alcázar, así como su conocida flema a la hora de pintar, que ya se empieza a manifestar, sorprendentemente, desde sus primeros años al servicio de Felipe IV.
\end{abstract}

Palabras clave: Francisco Barberini. Velázquez. Conde Duque Olivares. Pintura española. Siglo xviI.

New documents found in the Vatican Secret Archive concerning the shipment from Madrid to Rome of certain portraits painted by Velázquez for Cardinal Francesco Barberini are presented here. Among them is a preparatory drawing for a portrait of the Conde Duque de Olivares, to be painted according to the express indications left by the Cardinal and his secretary Cassiano del Pozzo in Madrid, in 1626. Also examined is the pontificial brief granted Velázquez in that year thanks to Barberini's intervention with the Pope, by which the painter could enjoy an ecclesiastical benefice even though he was a married layman with children. All of this information demonstrates the Conde Duque's interest in promoting the painter's career, the extraordinarily favorable treatment that the Sevillian received during his first years at the Alcázar, and his well-known slowness when it came time to paint, which — surprisingly - already manifested itself during this early period in the service of Felipe IV.

Key words: Francesco Barberini. Velázquez. Conde Duque de Olivares. Spanish painting. $17^{\text {th }}$ century.

* Los autores agradecen a Bill Jordan, Jonathan Brown, Edward Goldberg, Enriqueta Harris y Alfonso E. Pérez Sánchez, los valiosos consejos recibidos tras haber leído el primer borrador del texto. Asimismo se agradece a Janie Cole la revisión del texto en inglés.

AEA, LXXVII, 2004, 306, pp. 159 a 170 
«Havendo io pregato il sr. Cardinale Padrone si compiacese di lasciarsi ritratarsi a Gio. van der Gumen di Gualdarama (sic), spagnolo naturale di Madrid, che per fare ritratti, fiori e frutti e simili riusciva eccellentemente, havendo visto che il ritratto fatto da Gio. (sic) Velasquez per il sr. Conte d'Olivares riusciva d'aria maliconica e severa, si contentò che venisse, e in una mez'hora o poco più lo fece assai bene» ${ }^{1}$. Con estas conocidas palabras, Cassiano dal Pozzo expresaba su preferencia por el pintor Juan van der Hamen sobre Diego Velázquez, a la hora de hacer un retrato de su señor, el cardenal Francesco Barberini, cuando en 1626 ambos se encontraban en Madrid en misión extraordinaria ante Felipe IV. Tal vez sea ésta la única opinión negativa, jamás expresada, sobre la capacidad retratística del maestro sevillano a lo largo de su vida en la que, por otra parte, fue siempre considerado, en España e Italia, como uno de los genios absolutos en el género del retrato ${ }^{2}$.

En las siguientes páginas vamos estudiar dos consecuencias inmediatas sobre la vida y obra de Velázquez, que derivaron del viaje a Madrid realizado por los citados Barberini y Pozzo. De un lado el encargo a Velázquez, por parte del cardenal legado y a través de Giovanni Battista Pamphili, nuncio en Madrid, de unos retratos; entre ellos uno del propio conde duque, para el cual el prelado italiano había dejado órdenes expresas sobre su composición. Asimismo, vamos a estudiar la dispensa papal que Olivares había solicitado en Madrid a Barberini, para su pintor, con el objeto de que éste obtuviera una pensión eclesiástica de 300 ducados. Noticia que era conocida parcialmente, a través de Francisco Pacheco y que fue estudiada por otros dos investigadores, gracias a dos documentos al respecto encontrados en la Biblioteca Apostólica Vaticana. A estas informaciones ahora podemos añadir, por una parte, una carta de Barberini dirigida a Pamphili, en la que se explica la negativa inicial de Urbano VIII a conceder tal extraordinaria dispensa y, por otra, el breve pontificio - junto a su expediente- emitido finalmente por el papa, que completan la documentación hasta ahora conocida y ofrecen nuevos datos sobre el proceso.

Como es bien sabido, el cardenal Francesco Barberini (1597-1679), nipote de Urbano VIII y sovrintendente dello Stato della Chiesa, realizó, por orden del papa, una doble misión diplomática de máximo nivel como legato de latere ante Luis XIII y Felipe IV, con el objeto de poner fin a las hostilidades relacionadas con la Valtellina entre Francia y España, que se habían originado como consecuencia del «Sacro Macello» (19 de julio de 1620). Barberini, responsable, por lo menos nominalmente, de la política exterior pontificia, llevó a cabo su misión en Francia entre mayo y octubre de 1625, que concluyó sin ningún resultado. Como consecuencia de ello, el previsto viaje a España fue inicialmente cancelado por razones diplomáticas aunque, posteriormente, el prelado viajó a Madrid en 1626, con objetivos muy diferentes a los del problema de la Valltelina, ya que el tratado de Monzón, preparado entre Francia y España en secreto, se había firmado el 5 de marzo de aquel año. El nuevo objetivo del legado pontificio en la corte española era ahora el de apadrinar, en nombre del papa, a la infanta María Eugenia, que iba a ser bautizada. El cardenal llegó a Barcelona el 18 de marzo de 1626, abandonó Madrid el 12 de agosto y se embarcó en Valencia con rumbo a Italia el 24 de agosto. En este viaje le acompañaban el auditor de Rota Giovanni Battista Pamphili, futuro Inocencio X, que se quedó en España como nuncio ordinario, Lorenzo Azzolini, Antonio Santacroce y el erudito y coleccionista romano Cassiano dal Pozzo, que dejó una detallada descripción de aquel evento en su diario del viaje.

\footnotetext{
${ }^{1}$ Es un extracto del diario del viaje a España de Cassiano dal Pozzo que se conserva en la Biblioteca Apostólica Vaticana, Barb. lat. 5689, f. 133rv. Fue publicado por Enriqueta Harris, «Cassiano dal Pozzo on Diego Velázquez», The Burlington Magazine, 112, 1970, p.363-373. Asimismo, sobre el viaje, véase, Gregorio de Andrés y E. Harris, «Descripción de El Escorial por Cassiano dal Pozzo (1626)», en Archivo Español de Arte, vol. 45, 1972, anejo.

${ }^{2}$ Sobre la fortuna crítica de Velázquez en Italia véase S. Salort Pons, Velázquez en Italia, Madrid 2002, pp. $223-241$.
}

$A E A$, LXXVII, 2004, 306, pp. 159 a 170 
El diario de Cassiano, del que todavía no existe una edición crítica, es de máximo interés, no sólo para el estudio de las relaciones políticas durante el primer tercio del siglo XviI entre Madrid, París y Roma, sino también para el mejor conocimiento de la historia del arte y del coleccionismo españoles del Siglo de Oro ${ }^{3}$. A este último respecto, en el manuscrito se encuentran abundantes referencias sobre la decoración artística del Alcázar, así como algunos datos muy interesantes sobre Diego Velázquez. En este sentido, como Cassiano afirma, durante la estancia de la legación en Madrid, se tuvo la intención de realizar dos retratos, uno al conde duque y otro al cardenal, para que fueran intercambiados entre ambos ministros. En aquella época el mejor pintor del rey era Velázquez y, según dal Pozzo, el sevillano se encargó de pintar la imagen de Barberini, pero en su opinión este retrato hecho por el español resultó «d'aria malinconica e severa». Circunstancia que no le gustó y, en consecuencia, pidió que se repitiera el retrato de su señor encargándoselo, esta vez, a Juan van der Hamen.

Cuando la legación dejó Madrid, Olivares tenía en su poder, por lo menos, dos retratos del cardenal —uno de Velázquez y otro de van der Hamen-, sin embargo Barberini no se llevó a Roma el retrato que le correspondía del conde duque y, probablemente, ningún otro. Este hecho se deduce de tres cartas intercambiadas en los meses sucesivos entre el nuevo nuncio, Giovanni Battista Pamphili, y el propio cardenal. El 14 de octubre del 1626 (doc. 1) Pamphili informaba a Barberini de que, además de haber dado los debidos «complimenti» al rey y a su valido, iba a aprovechar la primera ocasión que tuviera para pedir a Olivares ciertos retratos y que, entre ellos, el del conde duque se iba a pintar de la forma en que el nipote del papa deseaba. El nuncio añade que «Sua Eccelenza» - refiriéndose al valido- por petición suya, había ya dado orden expresa al pintor ${ }^{4}$, que como veremos era Velázquez, de que los llevara a cabo.

Un mes más tarde Pamphili, junto al correo ordinario, mandaba un cilindro de metal que contenía el retrato de Olivares «de semplice dissegno», que al valido del rey le había parecido «bonissimo per l'effetto che si desidera» (doc. 2). Asimismo el nuncio expresaba su deseo de que el retrato estuviera de acuerdo con el gusto del propio Barberini y que, por lo que respectaba a los otros retratos, éstos no se habían terminado porque el pintor que los estaba haciendo «è molto flematico» ${ }^{5}$. El dibujo de la imagen de Olivares llegó a Roma en buenas condiciones, por lo que se refería al papel y a la figura, a pesar de que el cilindro de metal había sufrido desperfectos. En estos términos lo afirmaba el cardenal en una carta al nuncio del 31 de diciembre de 1626 (doc. 3), en la que además se mostraba plenamente satisfecho con la imagen e instaba al nuncio a que «riscaldara» con su «ardore» la flema del pintor ${ }^{6}$ para que, lógicamente, terminara los otros retratos y fueran enviados a Roma lo más pronto posible.

\footnotetext{
${ }^{3}$ Sobre las legaciones de Francesco Barberini en Francia y en España, véase: P. Blet, Histoire de la représentation diplomatique du Saint Siège des origines à l'aube du XIXe siècle (Collectanea Archivi Vaticani 9), Città del Vaticano 1982, p. 337-343; S. de Dainville-Barbiche, «Les légats 'a latere' en France et leurs facultés aux XVIe et XVIIe siècles», Archivum Historiae Pontificiae 23 (1985) p. 164-165; S. Giordano, «La Santa Sede e la Valtellina da Paolo V a Urbano VIII», en La Valtellina crocevia dell'Europa. Politica e religione nell'età della guerra dei trent'anni, dirección de A. Borromeo, Milano 1998, pp. 81-109; J. Simón Díaz, «La estancia del cardenal legado Francesco Barberini en Madrid el año 1626», Anales del Instituto de estudios madrileños 17 (1980) p. 159-213; J. Simón Díaz, «El viaje Madrid-Valencia del cardenal F. Barberini y su séquito (1626)», Studi di Iberistica in memoria di Giuseppe Carlo Rossi, Nápoles 1986, p. 198-213; M. del C. Simón Palmer, «Nuevos datos sobre el hospedaje del cardenal legado Francisco Barberini en Madrid el año 1626», Anales del Instituto de estudios madrileños 21 (1984) p. 411-434; A. Anselmi, «Il conflitto della Valtellina nel diario di Cassiano dal Pozzo (1626). I dipinti di Tiziano ai fini della propaganda politica nella Spagna di Filippo IV», La Valtellina crocevia dell'Europa. Politica e religione nell'età della guerra dei trent'anni, dirección de A. Borromeo, Milano 1998 pp. 219-231.

${ }^{4}$ BAV, Barb. lat., 8326. f. 105r. Se sabe que Barberini pagó 3 retratos a Van der Hamen. William B. Jordan, Spanish Still Life in the Golden Age, Fort Worth, Kimbell Art Museum, 1985, p. 124, n. 36.

${ }^{5}$ BAV, Barb. lat., 8326. f. 124r.

${ }^{6}$ ASV, Segreteria di Stato, Spagna, 343, f. 243v.
} 
No parece que se pueda dudar de que el pintor flemático, al que se refieren las cartas, sea el propio Velázquez y no Juan van der Hamen que, como el propio Cassiano afirma, había casi terminado el retrato de Barberini en poco más de media hora. La flema de Velázquez, a la hora de pintar, se repite en las fuentes españolas y también en las italianas a lo largo de la vida del pintor, después de su llegada a Madrid ${ }^{7}$. A este respecto, sorprende sin duda alguna el hecho de que Velázquez trabajara tan lentamente en sus primeros años en la corte, ya que su juventud y sus ambiciones palatinas lógicamente nos deberían inducir a pensar lo contrario. En todo caso, el maestro en esos años, y algunos documentos así lo demuestran, debió tener bastante trabajo a la hora de preparar retratos del valido y de los reyes para ser mandados a las cortes extranjeras ${ }^{8}$. Tal vez, por ello, el 19 de octubre de 1626, justo cuando se estaba llevando a cabo el encargo para Barberini, Velázquez recibió como aprendiz en su casa a Andrés de Brizuela ${ }^{9}$ para que, como es normal, le ayudara en las tareas menores de su trabajo.

Las cartas de Pamphili nos dan a entender que Barberini dejó órdenes expresas de cómo tenía que ser el retrato del conde duque. Esta hipótesis se puede confirmar con el hecho de que se enviara un dibujo preparatorio al prelado italiano con el objeto de que lo aprobase. Este requisito, quizás, fue motivado por consejo de Cassiano que, como hemos visto, no se sentía muy satisfecho por las capacidades retratísticas del sevillano. Si el coleccionista italiano pudo elegir el pintor - Juan van der Hamen - que realizara la imagen en lienzo de su señor, no parece que tuviera opción para elegir el artista que llevara a cabo el retrato del conde duque. Este hecho demuestra, a nuestro modo de ver, el protagonismo que tenía Velázquez sobre los otros pintores de corte y además prueba, de algún modo, las palabras de Francisco Pacheco, con las que afirma que el maestro tuvo el derecho exclusivo de pintar la imagen regia ${ }^{10} \mathrm{y}$, seguramente, también la del conde duque quien, como veremos más adelante, dejará entender, a través del nuncio, que Velázquez era su pintor.

Este dibujo se puede considerar, por lo tanto, como preparatorio para el retrato de Olivares, que Cassiano aseguraba que se iba pintar. Es uno de los pocos dibujos de los que se tiene certeza que realizó Velázquez y, junto al del retrato del cardenal Borja (Real Academia de San Fernando de Madrid), son los únicos dibujos preparatorios seguros para un retrato en pintura de un personaje hoy conocido ${ }^{11}$. Y por ello es del máximo interés, pues sabemos que el maestro no tenía la costumbre de dibujar antes lo que iba a pintar, tal y como demuestran las diversas correcciones $-\mathrm{o}$ arrepentimientos- que vemos en sus pinturas. Es posible pensar que la imagen de Olivares que tuvo Barberini fuera de cuerpo entero, pues en la carta se habla de la «figura» del ministro, y que esta «figura» pueda ponerse en relación - por lo que respecta a su tipología - con la del retrato del valido que, hoy en día, se conserva en la Hispanic Society de Nueva York, que para muchos especialistas se puede datar en torno a 1625 .

\footnotetext{
${ }^{7}$ La misma queja sobre la lentitud de Velázquez en su trabajo la manifestaron los embajadores de Módena y de Florencia en 1638 y 1639 respectivamente, cuando, por una parte, Francesco I pidió a Velázquez la copia de su retrato ecuestre y, por otra, cuando Giovan Carlo de Medici encargó unos retratos de los reyes de España al mejor pintor de la corte. Véase, S. Salort, obra cit. Madrid 2002, pp. 191-192.

${ }^{8}$ Por poner dos ejemplos: en 1626 el cardenal Sacchetti llevó a Roma tres retratos del taller de Velázquez y, un año más tarde, se mandaron unos retratos ecuestres del rey y de Olivares al duque de Mantua. Véase S. Salort, «Velázquez and Giulio Sacchetti: two unpublished portraits of the King and Queen of Spain», B.M., febrero 2001, pp. 67-72; A. Luzio, La galleria dei Gonzaga venduta all'Inghilterra nel 1627-28. Documenti degli Archivi di Mantova e Londra raccolti da Alessandro Luzio, Milano 1913, p. 56 y ss.

${ }^{9}$ F. Maldonado, «Episodio Velazqueño. Escritura inédita de asiento de un aprendiz», La Estafeta Literaria, n. 461, 1II-1971, pp. 4-7.

${ }^{10}$ Francisco Pacheco, El arte de la pintura, ed. Bassegoda i Hugas, Madrid 1990, p. 205.

${ }^{11}$ Sobre los dibujos de Inocencio X que se conservaban en la colección T.Allen Heinrich de Toronto, que en alguna ocasión se han puesto en relación con el retrato del papa, no hay unanimidad, por parte de los especialistas, sobre su paternidad velazqueña.
}

$A E A$, LXXVII, 2004, 306, pp. 159 a 170 
Asimismo no podemos dejar de recordar el dibujo, retrato del Conde Duque, atribuido a Velázquez, que se conserva en la École des Beaux Arts de París ${ }^{12}$. No hemos tenido la oportunidad de estudiar directamente la obra, pero parece evidente que la edad que demuestra el ministro en esta imagen correspondería aproximadamente a la que tenía cuando Barberini estuvo en Madrid. Igualmente es interesante observar que el tipo de composición con la que se representa, es decir vistiendo una armadura y con banda militar, recuerda al pequeño retrato de Felipe IV en el Museo del Prado hecho por Velázquez hacia 1625. Tal vez Barberini, aunque no lo podamos probar, quiso una imagen militar del valido, como algunos años más tarde, otro italiano, el duque de Módena, querrá su retrato con armadura y banda militar que, en este caso, era la de General de los Océanos. De hecho, se sabe que en el inventario de 1631 de la colección del cardenal de declara un «Un quadro con il ritratto del signor conte de Olivares, cioè testa e busto armato, di telaro di testa», del que, en todo caso, no se puede asegurar que sea la obra enviada por el nuncio Pamphili ${ }^{13}$.

Por lo que respecta a los otros retratos de los que se habla en las cartas, no podemos afirmar con seguridad de qué personajes se trataba ${ }^{14}$. No obstante es muy probable que fueran las imágenes del propio Felipe IV, la reina Isabel de Borbón y, tal vez, del infante don Carlos. Ello se puede deducir no sólo por cuestiones de lógica, sino también porque, por ejemplo, el nuncio Sacchetti en 1626 se había llevado de España retratos de esos miembros de la familia real hechos por el taller de Velázquez ${ }^{15}$. La diferencia entre las obras del recientemente nombrado cardenal Sacchetti y las del poderoso cardenal nipote, es que las de este último iban a ser pintadas directamente por la mano del propio Velázquez.

No hay motivos para pensar que el envío de estos retratos estuviera relacionado con la importante y difícil gestión que Barberini iba a realizar, desde Roma y en diciembre de 1626, para que concedieran a Velázquez una dispensa papal, con el objeto de que pudiera disfrutar de una pensión eclesiástica. En este sentido hay que recordar que Pacheco afirma que Felipe IV en 1626 dio a Velázquez 300 ducados de ayuda de costa y otros 300 ducados de una pensión eclesiástica como pago por el retrato ecuestre que había hecho del rey en ese tiempo.

Es seguro que Barberini y Cassiano admiraron el retrato ecuestre en el Alcázar, ya que este último nos ofrece una descripción del mismo en su diario ${ }^{16}$. No obstante en la documentación que se refiere al breve pontificio nunca se cita la existencia de tal retrato. La concesión de esta dispensa papal significaba el reconocimiento del rey de las dotes de su pintor, así como de la protección de la que Velázquez gozaba por parte de la familia real y su primer ministro. Por otra parte y desde el punto de vista eclesiástico, suponía una práctica absolutamente excepcional, por el hecho de que a los laicos no les era posible, por vías normales, el disfrute de un beneficio eclesiástico. Gracias a los trámites burocráticos que se llevaron a cabo y a la documentación que se conserva al respecto, es posible reconstruir con todo detalle la historia de esta excepcional concesión.

\footnotetext{
${ }^{12}$ López Rey lo excluye de su catálogo de las obras del maestro. Brown, sin embargo, la considera como posible dibujo de Velázquez. Un estudio de la procedencia de la obra podría ayudar a la hora de definir la paternidad velazqueña del dibujo. Véase, J. Brown, «A Portrait Drawing by Velázquez», Master Drawings, 14, 1976, pp. 46-51.

${ }^{13}$ J. Orbaan, Documenti sul barroco in Roma, Roma 1920, p. 508. Noticia que ya señaló Enriqueta Harris en, obra cit., 1970, pp. 363-373.

${ }^{14}$ Pamphili continuó informando al cardenal de otras noticias de carácter artístico como, por ejemplo, la llegada de Italia de una escultura en metal dorado para el conde duque: «Monsignor de Massimi con il galeone che di Napoli conduce quaranta cavalli al Re scrive di mandare al Conte Duca una Pietà di metallo dorata grande che puol servire per un altare, et a sua M.tà alcune galanterie. Intendo che il Conte Duca, havendo visto il disegno in tela, l'ha gradita per la Chiesa Collegiale che sta facendo in Siviglia. Ha però detto che vol sapere il costo, perché la vuol pagare». BAV, Barb. lat. 8330, f. 53. Madrid, 11 de septiembre de 1627.

${ }^{15}$ S. Salort, obra cit., B. M., febrero 2001, p. 67-72.

${ }^{16}$ BAV, Barb. lat. 5689, f. 48rv. Cfr. E. Harris, obra cit., B. M. 112, 1970, p. 363-373.
} 
En 1981 Enriqueta Harris ${ }^{17}$ publicó el memorial dirigido al cardenal Barberini, referido a Velázquez, por el que el pintor solicitaba la dispensa pontificia necesaria para poder gozar de la pensión eclesiástica que le concedió Felipe IV. El documento había sido enviado a Roma por el nuncio Pamphili junto a la correspondencia ordinaria del 14 de octubre de $1626^{18}$ (doc. 4). El texto en cuestión es una copia; el memorial, escrito originalmente en español, presenta algunos errores debido, evidentemente, a la no familiaridad del copista romano con la lengua. En un estudio de $1984{ }^{19}$, Quintín Aldea Vaquero publicó el memorial original, también conservado en la Biblioteca Apostólica Vaticana ${ }^{20}$, y una carta del nuncio en el que se hace referencia al citado memorial ${ }^{21}$ (doc. 5). No obstante, no se conocía la concesión de la dispensa por parte del pontífice. Dos recientes descubrimientos en el Archivo Secreto Vaticano nos permiten recuperar el eslabón de la cadena que faltaba, así como esclarecer las difíciles circunstancias en las que la dispensa fue emitida, completando, de este modo, la historia de la concesión.

Se trata, por una parte, de un carta del Francesco Barberini al nuncio Pamphili en la que se explica la negativa del papa para llevar a cabo la extraordinaria dispensa y, por otra, del propio breve pontificio ${ }^{22}$ en el que se contienen: la súplica original, sin fecha, escrita en italiano y dirigida al papa, tal y como se acostumbraba, en la que se refiere parte de lo que había declarado Velázquez en su memorial dirigido al cardenal (doc. 8); una carta original de Barberini a Ulpiano Volpi, secretario de breves (1623-1627), con la que el cardenal le solicitaba «negotiare questa gratia con la Santità Sua» (doc. 7); y finalmente la minuta del breve con el que el papa concedía a Velázquez el derecho de poder percibir la pensión eclesiástica, no obstante su condición de laico casado (doc. 9).

La tramitación de la «grazia» empezó en España, durante la legación de Barberini «Mentre io ero legato in Ispagna, fui costretto di promettere al alcuni dei quei Signori Grandi d'impetrare gratia dalla Santità di Nostro Signore per Diego Vasquez [sic] pittore del re di poter tenere trecento ducati di quella moneta di pensione, non ostante ch'egli sia clerico coniugato» ${ }^{23}$ (doc. 7) . La promesa es recordada a Barberini mediante el memorial de Velázquez que Pamphili envía al cardenal, junto a la correspondencia ordinaria del 14 de octubre de $1626^{24}$. El memorial está dirigido a Francesco Barberini en calidad de legado de latere, y probablemente se le habría de haber entregado durante su estancia en Madrid. A este respecto es significativa la siguiente frase: «A V. S.a Illtm.a suplica, pues tiene en esta parte sus vezes, le haga merced de dispensar con el», en la que «esta parte» indica el lugar en el que fue escrito el memorial, es decir España, y «sus vezes» se refiere a la función de legado de latere, que constituía el grado más alto de representación del papa ${ }^{25}$. El memorial está dividido en dos partes, en primer lugar recuerda el «status» de pintor de Su Majestad del solicitante, su condición de hombre casado y el hecho de que el rey le había concedido, o por lo menos, prometido

\footnotetext{
${ }^{17}$ E. Harris, «Velázquez and his Ecclesiastical Benefice», B. M. 123, 1981, p. 95-96.

${ }^{18}$ BAV, Barb. lat. 6125, f. 320rv, copia.

${ }^{19} \mathrm{La}$ noticia en primer lugar la publicó Camón Aznar en su libro sobre Velázquez señalando, en todo caso, que el descubrimiento se debía a Quintín Aldea. Q. Aldea, «Diego Velázquez pensionado eclesiástico por Canarias», Studia historica et philologica in honorem M. Battlori, Roma 1984, p. 33-40. Un breve apunte sobre la cuestión lo encontramos en Q. Aldea, «La economía de las iglesias locales en la edad media y moderna», Hispania Sacra 26 (1973) p. 39-40. Q. Aldea no parece que conociera el citado trabajo de Harris al respecto.

${ }^{20}$ BAV, Barb. lat. 8326 , n. 99 , original, sin fecha. No parece autógrafo de Velázquez.

${ }^{21}$ BAV, Barb. lat. 8326, n. 98: Giovanni Battista Pamphili al cardenal Francesco Barberini, Madrid, 14 de octubre de 1626 , original.

${ }^{22}$ ASV, Sec. Brev., Reg. 720, f. 432r-436v.

${ }^{23}$ ASV, Sec. Brev., Reg. 720, f. 433r: Francesco Barberini a Ulpiano Volpi, Roma, 17 de diciembre de 1626, original.

${ }^{24}$ BAV, Barb. lat. 8326, n. 99: Giovanni Battista Pamphili a Francesco Barberini, Madrid, 14 de octubre de 1626, original.

${ }^{25}$ Según Q. Aldea, obra cit., Studia historica et philologica in honorem M. Batllori, Roma 1984, p. 38, el memorial debió estar dirigido al nuncio. Éste, sin embargo, no tenía tan amplios poderes de dispensa, que estaban reservados a la Santa Sede.
}

$A E A$, LXXVII, 2004, 306, pp. 159 a 170 
la pensión, para la que era necesaria la dispensa pontificia. En la segunda parte, se pide formalmente la dispensa y se recuerda la imposibilidad del caso en cuestión, ya que Velázquez tenía sólo dos hijas, de no poder recurrir a un expediente frecuentemente puesto en acto en circunstancias similares: es decir el conceder la pensión a un hijo varón, generalmente menor de edad, por lo tanto célibe, al que en esta ocasión se le imponía la tonsura ${ }^{26}$. Esta permitía acceder de modo formal al estado eclesiástico y, por lo tanto, de gozar de los correspondientes beneficios eclesiásticos, pero no conllevaba obligaciones gravosas, como la renuncia al matrimonio, que sólo era obligatoria con la ordenación subdiaconal.

La carta de acompañamiento de Pamphili ${ }^{27}$ revela la identidad de la persona que intercedió por Velázquez: «Fra l'altre gratie delle quali presuppone il s. Conte Duca haver qui supplicato V. S. Ill.ma acciò restasse servita impetrargliele da Sua Santità, m'ha detto esserne una la contenuta nell'aggiunta nota». Según la misiva del nuncio, el rey presionaba para que la gracia fuera concedida y Olivares insistía al nuncio en beneficio «del suo pittore» (doc. 4).

La cuestión fue elevada al juicio de Urbano VIII el 17 de diciembre del 1626 por el datario Egidio Orsini de Vivariis (1626-1644) ${ }^{28}$, pero el papa dio una respuesta negativa. Según un nota en el reverso de la carta, «S. Stà anco non inclina, e da qui inanzi si dichiara di non volere concedere simili gratie». Vista la situación, Barberini, inmediatamente informado de la negativa, escribió el mismo día dos cartas: una dirigida al nuncio en Madrid y otra a Ulpiano Volpi, secretario de breves. La primera refiere lo siguiente: «Havendo concessa S. S.tà altra unione de benefici liberi ad instanza del sr. Conte Duca e sua signora consorte, sopra di che interposi ancor io le mie preghiere con S. B. mentre dimoravo costì, non ho potuto poi cavarne altra simil gratia sul contenuto della nota mandatami da V. S. con lettera de 14 ottobre, sì come ne meno è parso alla S.tà S. di dispensare il pittor Diego Velazquez, che ha moglie e figli, a ritenere pensione ecclesiastica, havendo di già la $S$.tà $S$. fatto proponimento per l'esemplarità e conseguenze di tali concessioni di non condescendervi più per l'avvenire. Potrà però V. S., col dar certezza a S. E. del vivo affetto con che son tornato a supplicar S. S.tà delle stesse gratie, renderla insieme capace delle ragioni che astringono S. B. a non moltiplicare, et assicurandola di più, ch'il mio presente sentimento di non haver potuto...» ${ }^{29}$ (doc. 6).

En la carta a Volpi, Barberini le ruega que intervenga ante el papa: «è necessario che V. S. $\mathrm{mi}$ faccia piacere di negotiare questa gratia con la Santità Sua e procurare in ogni maniera ch'io sodisfaccia alla mia promessa» ${ }^{30}$ (doc. 7). A pesar de que el cardenal, como él mismo explica en su carta al nuncio, ya había conseguido algunos beneficios extraordinarios para el conde duque y su consorte durante su estancia en Madrid y el papa se estaba cansando de este tipo de concesiones, Volpi fue eficaz. Éste hizo preparar una súplica ${ }^{31}$ (doc. 8) mucho más sintética que el memorial que había presentado Velázquez a Barberini, en la que se pedía exclusivamente la dispensa para poder «tenere la ditta pensione, non ostante che sia maritato». El relativo breve fue expedido el 23 de diciembre de 1626, y es incluso más amplio que la petición en sí, pues se concedió a Velázquez la posibilidad de poder gozar de una o más pensiones hasta un máximo de 300 ducados sobre cualquier beneficio eclesiástico.

La pensión eclesiástica se define como el derecho concedido por la autoridad eclesiástica para percibir cada año una parte de las rentas de un beneficio del que no se es titular, sin

\footnotetext{
${ }^{26}$ No necesariamente, como parece suponer Harris, obra cit., B. M. 123, 1981, p. 95, los hijos de artistas o de otros personajes a los que se adjudicaba una pensión, administrada por sus padres, recibían las órdenes sagradas.

${ }^{27}$ BAV, Barb. lat. 8326, n. 98: Pamphili a Barberini, Madrid, 14 de octubre de 1626, original.

${ }^{28}$ A. Kraus, Das päpstliche Staatssekretariat unter Urban VIII. 1623-1644 (Römische Quartalschrift für christliche Altertumskunde und Kirchengeschichte 29), Rom-Freiburg-Wien 1964, p. 39, 276, 303.

${ }^{29}$ ASV, Segr. Stato, Spagna, 343, f. 239rv.

${ }^{30}$ ASV, Sec. Brev., Reg. 720, f. 433r, Barberini a Volpi, Roma, 17 de diciembre de 1626, original.

${ }^{31}$ ASV, Sec. Brev., Reg. 720, f. 434r, original.
} 
tener por ello que dar a cambio alguna prestación. Ésta se denomina personal cuando grava sobre el beneficiario y cesa con su muerte, mientras que se denomina real cuando grava sobre el beneficio y se debe pagar por todos los sucesivos beneficiarios hasta la muerte del pensionado ${ }^{32}$.

En España, debido a los derechos de patronato concedidos por los papas a la Corona en el primer tercio del siglo XvI, el rey tenía el derecho de conceder pensiones sobre los beneficios eclesiásticos. La tramitación seguía tres etapas: la concesión, que venía efectuada generalmente mediante una cédula real que indicaba la cantidad de la suma concedida, la «situación», es decir la indicación del beneficio sobre el que se tenía que recibir las rentas y, finalmente, el pago en sí. Estas tres etapas no eran necesariamente consecutivas: no era extraño que una pensión concedida pudiera esperar años antes de ser «situada». De hecho, ello no sucedía si no en el momento en el que un beneficio quedaba vacante por muerte o por cambio del titular y una parte de su renta nominal venía asignada a uno o más pensionados indicados por el rey. Por último, el pago efectivo de la suma se llevaba a cabo en base a las condiciones reales del beneficio eclesiástico, que variaban según la coyuntura económica.

A Velázquez la pensión le fue «situada» por voluntad de Felipe IV unos seis meses más tarde de la concesión de la dispensa pontificia: con una orden del 8 de julio de 1627, el rey indicó el importe de la pensión, correspondiente a la suma de 300 ducados, que se debían pagar de las rentas de la diócesis de Canarias. El pago tenía que efectuarse a partir del momento en que el papa hubiera nombrado, como nuevo obispo, al prelado presentado por el rey, Cristóbal de la Cámara y Murga ${ }^{33}$. El candidato, nativo de la diócesis de Burgos, era canónigo magistral de la metropolitana de Toledo y vicario general de aquel arzobispo. Fue nombrado por el rey obispo de Canarias el 26 de junio de 1627 y fue confirmado por Urbano VIII el 15 de noviembre del mismo año. La renta episcopal de las islas tenía un valor nominal anual de 20.000 ducados y, en ocasión del nuevo nombramiento, se reservaron 4.200 para las pensiones, incluyendo las que ya habían sido asignadas precedentemente y que correspondían a un quinto del total ${ }^{34}$. ¿El pintor recibió realmente la pensión? Solamente una investigación en los registros contables diocesanos de Canarias podría ofrecer datos en relación con el posible pago efectivo y sus modalidades.

La inscriptio del breve se refiere a Velázquez como «clerico coniugato». La entrada en el estado clerical se realizaba mediante la tonsura, que era un simbólico corte de pelo y barba; de ella derivaban privilegios y exenciones. Los clérigos tenían derecho a percibir los diezmos y recibir beneficios eclesiásticos; estaban exentos de servicio militar, ya que no tenían el derecho de llevar armas, mientras que las acciones violentas que pudieran sufrir venían castigadas, por el canon 15 del concilio Lateranense II (1139), con la excomunión reservada al papa. Debido a esta situación, aquéllos quedaban exentos del poder coercitivo de los jueces laicos (privilegium fori) ${ }^{35}$.

El Concilio de Trento hace referencia a los clérigos casados ${ }^{36}$, obviamente no ordenados in sacris, a los que el obispo podía delegar un servicio o un ministerio en la iglesia, con la obligación de llevar el hábito clerical y la tonsura. El canon en cuestión, para definir el estatu-

\footnotetext{
${ }^{32}$ R. Naz, «Pension ecclésiastique», en Dictionnaire de Droit Canonique, 6 Paris 1957, col. 1346-1353.

${ }^{33}$ Q. Aldea, obra cit., Studia historica et philologica in honorem M. Battlori, Roma 1984, p. 39.

${ }^{34} \mathrm{P}$. Gauchat, Hierarchia Catholica medii et recentioris aevi, IV, Monasterii 1935, p. 132. Cristóbal de la Cámara sucedió a Juan de Guzmán, que a su vez fue transferido a la sede arzobispal de Tarragona.

${ }^{35}$ R. Naz, «Tonsure», en Dictionnaire de Droit Canonique, 7, Paris 1965, col. 1289-1293.

${ }^{36}$ Concilium Tridentinum, sessio XXIII, (15 de julio de 1563) de reformatione, can. 6, in Conciliorum Oecumenicorum Decreta, edición de G. Alberigo - G. L. Dossetti - P.-P. Joannou - P. Prodi, Bologna 1991, p. 747; C. Lefebvre, «Le système bénéficial», en J. Bernhard - C. Lefebvre - F. Rapp, L'époque le la Réforme et du concile de Trente (Histoire du droit et des institutions de l'Église en Occident 14), Paris 1989, p. 361.
}

$A E A$, LXXVII, 2004, 306, pp. 159 a 170 
to de la categoría, hace referencia a la constitución Clerici, qui cum unicis ${ }^{37}$ del papa Bonifacio VIII (1294 - 1303), contenida en el «Liber sextus decretalium», según la cual sólo el clérigo casado que llevara la tonsura y el hábito propio de su estado tenía derecho de gozar de los privilegios clericales.

Mediante el breve concedido por Urbano VIII, Velázquez fue dispensado de llevar el hábito y por ello podía gozar sólo de la pensión y no del privilegio del foro. ¿Dónde y cuándo el pintor recibió la tonsura? Ésta era concedida por el obispo diocesano o por el superior regular. El breve pontificio no ofrece indicaciones precisas sobre la diócesis a la que pertenecía y, por ello, tampoco del obispo que se la pudo conferir. La indicación de la inscriptio, «clerigo coniugato Toletanae seu alterius diocesis» es voluntariamente imprecisa, ya que en ella parece que se supone que Velázquez, presente en Madrid, pudiera pertenecer a la diócesis de Toledo, como también a cualquier otra. Por otra parte, la continua oscilación de la terminología en los diferentes documentos — se habla de «laico coniugato», «clerico coniugato», «tenere la pensione non ostante che sia maritato»— nos haría más bien suponer que Velázquez no llegó a recibir nunca la tonsura. De hecho el término «clericus coniugatus» aparece solamente en los documentos de la cancillería pontificia, preocupada, naturalmente, en la corrección formal de los documentos emitidos, como también del hecho de que, como consecuencia de una concesión de una gracia extraordinaria, no se creara un precedente tal, que diera a los laicos la vía libre de acceso a los privilegios del estado clerical.

No hay duda de que Velázquez consiguió su beneficio eclesiástico gracias a la habilidad del cardenal Barberini y sus contactos en Roma. El rey y el conde duque presionaron al nuncio, para que el cardenal se empleara al máximo de sus posibilidades, pues como el propio prelado afirma fue obligado a prometer en España a «quei SS.ri Grandi», el conceder la difícil «gracia» al pintor de Su Majestad. Velázquez ya tenía una gran influencia en palacio, de hecho el 7 de marzo de 1627, era nombrado Ugier de Cámara y, como afirmará el embajador florentino en Madrid en 1629, Averardo de Medici, «prattica molto adentro in Corte». Felipe IV lo premió en gran medida con los 300 ducados de la pensión y con los otros 300 de ayuda de costa, si consideramos que su salario mensual era de 20 ducados, más el pago de cada obra realizada. Ningún otro artista de palacio había recibido tanto favor y, con ello, se demuestra el protagonismo del joven sevillano en la corte, en la que era el primer pintor de Felipe IV y de Olivares, a pesar de su conocida flema y de las preferencias de Cassiano. Por esta razón el retrato del conde duque y los otros lienzos mandados a Barberini fueron pintados por Velázquez y no por Juan van der Hamen. El cardenal Barberini seguirá en los años siguientes favoreciendo al «protegé» del Alcázar cuando en 1630 el artista llegue a Roma y sea alojado nada menos que en los palacios vaticanos, donde pudo estudiar las obras de Rafael y Miguel Ángel con plena libertad. Desconocemos si entonces Velázquez, en agradecimiento, pintó algún cuadro para el cardenal, que atesoraba una de las colecciones más importantes de Roma y en la que se conservaba, por lo menos, el dibujo del conde duque, y seguramente los otros retratos. Velázquez había entrado en Italia con sus obras, y en la colección de uno de los mecenas más importantes de la primera mitad del siglo xvII, protector de Poussin, Reni, Lanfranco, Cortona, y Sacchi, de los que tanto el genial sevillano iba a aprender.

\footnotetext{
${ }^{37}$ Bonifacius VIII, «Liber sextus decretalium» lib. III, tit. II, cap. I, en Corpus Iuris canonici, edición de Ae. Friedberg, Graz 1959, col. 1019.
}

AEA, LXXVII, 2004, 306, pp. 159 a 170 


\section{APÉNDICE DOCUMENTAL}

1. Carta de Giovanni Battista Pamphili a Francesco Barberini, Madrid, de 14 octubre de 1626. Original BAV, Barb. lat. 8326. f. 105r.

....Ho passato il dovuto complimento con il sr. Conte Duca e le ne rende infinite gratie, mostrandomi poi esserle stata estraordinariamente cara la nuova della sua salute. Farò l'istesso con S. M.tà con la prima occasione. Intanto sollicitarò li ritratti, e quello in particolare del sr. Conte si farà nella forma che V. S. Ill.ma commanda, e già S. E. a mia instanza ne ha dato ordine espresso al Pittore.....

2. Carta de Giovanni Battista Pamphili a Francesco Barberini, Madrid, 16 de noviembre de 1626. Original

BAV, Barb. lat. 8326. f. 124r.

In un canello de latta che invio con questo ordinario, riceverà V. S. Ill.ma. il retratto del sr. Conde Duca de semplice desegno. Ho voluto che l'istesso sr. Conte lo veda, e li è parso bonissimo per l'effetto che si desidera, così desiderarei che riuscisse a V. S. Ill.ma per la passione che tengo de incontrare in qualsiasivoglia cosa il suo gusto, Gli altri retratti si vano faccendo, ma in vero questo pittore è molto flematico, con che per fine faccio a V. S. Ill.ma humilissima riverenza. Madrid 17 novembre 1626.

Humilissimo et obligatissimo servitore. G. B. Patriarca de Antiochia.

3. Carta de Francesco Barberini a Giovanni Battista Pamphili, Roma, 31 de diciembre de 1626. ASV, Segr. Stato, Spagna, 343, f. 243v, copia.

Il canello di latta col ritrato del sr. Conte Duca di San Lucar ricevuto col ordinario insieme con una lettera di V. S. de 17 cadente, benché fusse alquanto schiacciato, non ha guasto in parte alcuna la carta né la figura, la quale essendo di mia piena sodisfattione, ne ringratio l'amorevollezza di lei. Per gli altri ritratti, se bene il Pittore è flemmatico, lo riscalderà l'ardore di V. S., alla quale prego dal cielo ogni contentezza.

4. Carta de Giovanni Battista Pamphili a Francesco Barberini, Madrid. 14 de octubre de 1626.

BAV, Barb. lat. 8326, n. 98, original; Barb. lat. 6125, f. 320rv, copia.

Publicado (sólo el post-escrito autógrafo): E. Harris, «Velázquez and his Ecclesiastical Benefice», en The Burlington Magazine 123 (1981), p. 96 (ex Barb. lat. 6125); Q. Aldea, «Diego Velázquez pensionado eclesiástico por Canarias», Studia historica et philologica in honorem M. Battlori, Roma 1984, p. 39 (ex Barb. lat. 8326).

Ill.mo e R.mo S.r mio P.ron Col.mo

Fra l'altre gratie delle quali presuppone il s. Conte Duca haver qui supplicato V. S. Ill.ma acciò restasse servita impetrargliele da Sua Santità, m'ha detto esserne una la contenuta nell' aggiunta nota, in cui mostra premere assaissimo la Maestà del Re. M'ha pregato che io ne rinfreschi la memoria a V. S. Ill.ma et in suo nome la supplichi di nuovo efficacemente, il che havendo con questa essequito, le faccio humilissima riverenza.

Di Madrid 14 ottobre 1626

Di V. S. Ill.ma e R.ma

all S.r Conte Duca mi ha anco pregato che invii l'aggiunto memoriale a V. S. Ill.ma che è del suo pittore. Humiliss.o et oblig.mo ser.re

G. B. Patriarca de Antiochia ${ }^{1}$

en el verso del original

1626

Di Madrid 14 ottobre

Mons.r Nunzio

N.ro Sig.re non intende di concedere l'incluse gratie havendo già concesso unioni di beneficii liberi ad instanza delli SS.ri Conde Condessa d'Olivares per alcune migliaia di ducati.

Per conto del Patrone

S. S.tà anco non inclina et da qui inanzi si dichiara di non volere concedere simili gratie

A Mons. Dat(ari)o con N. S. e col S. Card.le

R.to a 17 decembris

${ }^{1}$ Autógrafo del nuncio.

$A E A$, LXXVII, 2004, 306, pp. 159 a 170 
5. Memorial de Diego Velázquez a Francesco Barberini, sin fecha.

BAV, Barb. lat. 8326, n. 99, original; Barb. lat. 6125, fol. 320rv, copia.

Publicado en: E. Harris, «Velázquez and his Ecclesiastical Benefice», en The Burlington Magazine 123 (1981), p. 96 (ex Barb. lat. 6125); Q. Aldea, «Diego Velázquez pensionado eclesiástico por Canarias», Studia historica et philologica in honorem M. Battlori, Roma 1984, p. 38 (ex Barb. lat. 8326).

\section{Illtm.o i R.mo Señor}

Diego Velasquez, pintor de Su Mag.tad, dize que, atento a que es pobre i tiene muger i hijas, Su Mag.tad le a hecho merced de darle trezientos ducados de pension cada año en moneda de Castilla sobre beneficios eclesiasticos u obispados; i siendo como es casado no los puede gozar sin particular dispensacion para ello de Su Santidad.

A V. S.a Illtm.a suplica, pues tiene en esta parte sus vezes, le haga merced de dispensar con el, atento que no tiene hijo varon en cabeça de quien pueda poner la dicha pension, que esto mesmo an hecho los Pontifices de beata memoria que an passado con otros muchos que, siendo casados, gozan oi pensiones eclesiasticas. Que en ello recebira merced de la mano de V. S.a Illtm.a.

\section{verso \\ Illtm.o i R.mo Señor \\ Diego Velasquez}

Pintor de Su Mag.tad

6. Carta de Francesco Barberini a Giovanni Battista Pamphili, Roma, 17 de diciembre de 1626.

ASV, Segr. Stato, Spagna, 343, f. 239rv, copia.

Havendo concessa S.Sta. altra unione de Benefici liberi ad instanza del sr. Conte Duca, e sua signora consorte, sopra di che interposi ancor io le mie preghiere con S. B. mentre dimoravo costì, non ho potuto poi cavarne altra simil gratia sul contenuto della nota mandatami da V. S. con lettera de 14 ottobre, sì come ne meno è parso alla S.tà $S$. di dispensare il pittor Diego Velazquez, che ha moglie e figli, a ritenere pensione ecclesiastica, havendo di già la S.tà S. fatto proponimento per l'esemplarità e conseguenze di tali concessioni di non condescendervi più per l'avvenire. Potra però V. S. col dar certezza a $\mathrm{S}$. E. del vivo affetto con che son tornato a supplicar S. S.tà delle stesse gratie, renderla insieme capace delle ragioni che astringono S. B. a non moltiplicare, et assicurandola di più, ch'il mio presente sentimento di non haver potuto servire a mio e suo gusto l'E. S. mi fa impatiente d'altre occasioni da ricompensar questa col sodisfare a i suoi commandamenti, et a V.S. per finire mi offro.

7. Carta de Francesco Barberini a Ulpiano Volpi, Roma, 17 de diciembre de 1626.

ASV, Sec. Brev., Reg. 720, f. 433r, original.

Molt'Ill.re et R.mo S.r come fratello. Mentre io ero legato in Ispagna, fui costretto di promettere ad alcuni di quei SS.ri Grandi d'impetrare gratia dalla Santità di N. S.re per Diego Vasquez, pittore del re, di poter tenere trecento ducati di quella moneta di pensione, non ostante ch'egli sia clerico coniugato. E però è necessario che V. S. mi faccia piacere di negotiare questa gratia con la Santità Sua e procurare in ogni maniera ch'io sodisfaccia alla mia promessa e che quell'huomo ne venga consolato. E per fine a V. S. prego da Dio ogni contento.

Di Palazzo a 17 di decembre 1626.

D V. S. I.

Come fratello aff.mo

F. Card. Barberino ${ }^{2}$

8. Súplica en favor de Diego Velázquez, sin fecha.

ASV, Sec. Brev., Reg. 720, f. 434r-436v, original.

[f. 434r]

Beatissimo Padre

Diego Velasguez pittore del Re Catolico humilissimo servo di V. B., havendo ottenuto da S. M. la gratia per trecento ducati di pensione ecclesiastica, humilmente supplica che V. S. le voglia fare un breve per pote-

\footnotetext{
2 Signatura autógrafa.
} 
re tenere la ditta pensione, non ostante che sia maritato; e di tal gratia restarà obligatissimo di pregare la divina Maestà per la conservation di V. S. Quam Deus.

\section{[f. 435v]}

Alla Santità di N.ro Sig.re

Il sig. Card.le Barberino che quanto prima si spedischi

Pro Diego Velasquez pittore del Re Catolico

\section{[f. 436v]}

Pro Didaco Vasquez clerico coniugato

Indultum obtinendi pensiones pro summa 300 ducatorum

habuit Ill.mus D. Card.is Barberinus

\section{Urbano VIII concede a Diego Velázquez, pittore del re Cattolico, gozar una pensión eclesiástica de} 300 ducados, aun siendo casado.

ASV, Sec. Brev., Reg. 720, f. 432rv, minuta.

[f. 432r]

Dilecto filio Didaco Velasquez clerico coniugato Toletanae seu alterius diocesis Urbanus Papa VIIIs

Dilecte. \&. Sincere \& opportunum.

Volentes igitur tibi qui, ut asseris, charissimi in Christo filii nostri Philippi Hispaniarum Regis Catholici pictor existis premissorum meritorum tuorum intuitu gratiam facere specialem teque a quibusvis \& censentes, supplicationibus tuo nomine inclinati, tecum ut, etiam si coniugatus existas, nihilhominus unam seu plures pensiones annuas usque ad summam trecentorum ducatorum monete Hispaniarum ${ }^{3}$ super quibusvis fructibus ecclesiasticis apostolica tibi auctoritate reservandas obtinere ac percipere exigere et levare ac in tuos usus et utilitatem convertere libere et licite valeas apostolica auctoritate tenore presentium dispensamus, tibique concedimus et indulgemus. Decernentes pensionum huiusmodi debitores ad illarum solutionem debito tempore tibi faciendam iuxta illarum reservationum formam et tenore integre faciendam omnino teneri et obligatos existere sicque per quoscumque iudices \& auditores iudicari [f. 432v] attentari. Non obstantibus pie memorie Pii Pape V predecessoris nostri ${ }^{4}$ ac aliis apostolicis nec non in universalibus conciliis editis generalibus vel specialibus constitutionibus et ordinationibus, ceterisque contrariis quibuscumque.

Datum Rome apud S. Petrum die 23 decembris 1626 anno IV.

Pro pictore Regis Catholici. Indultum obtinendi pensiones pro summa 300 ducatorum licet sit coniugatus etiam absque habitus susceptione ${ }^{5}$.

Ill.mo Card. Barberino Intercedente ${ }^{6}$

$\mathrm{Ph}$ (ilippus) $\mathrm{M}$.

C

V. Theatin. ?

\footnotetext{
3 auri de Camera del.

${ }^{4}$ Pio V, constitución Ex proximo, 20 settembre 1571, en Bullarium Romanum, 7, Neapoli 1882, pp. 942-943. Establece algunas penas para aquellos que, gozando de los beneficios simples o con cura animarum no rezan las horas canónicas, y obliga a todos los que poseen beneficios eclesiásdticos de rezar el Officium parvum beatae virginis Mariae.

5 Otra mano.

${ }^{6}$ Tercera mano.

7 Ulpiano Volpi, arzobispo de Gieti (1609-1615); obispo de Novara (1619-1622). El papa le concedió eld erecho de conservar la denominación de su primera sede obispal. P. Gauchat, Hierarchia Catholica, IV, cit., pp. 262, 332.
}

AEA, LXXVII, 2004, 306, pp. 159 a 170 